Original article

\title{
Non-alcoholic fatty liver in hereditary fructose intolerance
}

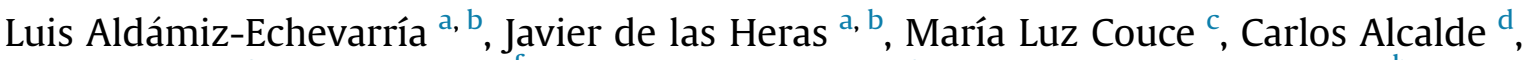 \\ Isidro Vitoria ${ }^{\mathrm{e}}$, María Bueno ${ }^{\mathrm{f}}$, Javier Blasco-Alonso ${ }^{\mathrm{g}}$, María Concepción García ${ }^{\mathrm{h}}$, \\ Mónica Ruiz ${ }^{\mathrm{i}}$, Rafael Suárez ${ }^{a}$, Fernando Andrade ${ }^{\mathrm{a}}$, Olatz Villate ${ }^{\mathrm{a}, *}$ \\ ${ }^{a}$ Unit of Metabolism, Cruces University Hospital, Biocruces Bizkaia Health Research Institute, GCV-CIBER de Enfermedades Raras (CIBERER), Plaza de Cruces \\ $s / n$, Barakaldo, 48903, Spain \\ ${ }^{\mathrm{b}}$ University of the Basque Country (UPV/EHU), Metab-European Reference Network, Spain \\ ${ }^{\mathrm{c}}$ Unit of Diagnosis and Treatment of Congenital Metabolic Diseases, Department of Pediatrics, Hospital Clínico Universitario de Santiago de Compostela, \\ CIBERER, Health Research Institute of Santiago de Compostela (IDIS), A Choupana, s/n, Santiago de Compostela, A Coruña, 15706, Spain \\ ${ }^{\mathrm{d}}$ Paediatrics Unit, Río Hortega University Hospital, Calle Dulzaina 2, Valladolid, 47012, Spain \\ e Unit of Metabolopathies, La Fe University Hospital, Valencia, 46026, Spain \\ ${ }^{\mathrm{f}}$ Metabolic Disorders, Dietetics and Nutrition Unit, Virgen del Rocío University Hospital, Manuel Siurot Avenue, s/n, Sevilla, 41013, Spain \\ ${ }^{g}$ Pediatric Gastroenterology, Hepatology and Nutrition Unit, Hospital Regional Universitario de Málaga, Málaga, 29001, Spain \\ h Metabolic Pathologies Unit, Miguel Servet University Hospital, Zaragoza, 50009, Spain \\ i Paediatrics Unit, Nuestra Señora de la Candelaria University Hospital, Tenerife, 38010, Spain
}

\section{A R T I C L E I N F O}

\section{Article history:}

Received 18 September 2018

Accepted 10 February 2019

\section{Keywords:}

Non-alcoholic fatty liver disease Hereditary fructose intolerance Mutation

ALDOB gene

\begin{abstract}
S U M M A R Y
Background: Non-alcoholic fatty liver disease (NAFLD) is characterized by fat accumulation affecting $>5 \%$ of the liver volume that is not explained by alcohol abuse. It is known that fructose gives rise to NAFLD and it has been recently described that the ingestion of fructose in low amounts in aldolase B deficient mice is associated with the development of fatty liver. Therefore, it is reasonable that patients with HFI (Hereditary Fructose Intolerance) present fatty liver at diagnosis, but its prevalence in patients treated and with adequate follow-up is not well documented in the literature. The aim of this study is to analyze the association between HFI and NAFLD in treated patients.

Methods: A cross-sectional observational study was conducted. The population comprised 16 genetically diagnosed HFI patients aged from 3 years to 48 and in dietary treatment of fructose, sorbitol and sacarose exclusion at least for two years. Blood samples were obtained for analytical studies and anthropometric measurements of each patient were performed.

Results: Patients presented a Body Mass Index (BMI) of $17.9 \pm 2.9 \mathrm{~kg} / \mathrm{m}^{2}$. The HOMA index and Quick index were in normal range for our population. The S-adenosyl-methionine (SAM)/S-adenosyl-Lhomocysteine $(\mathrm{SAH})$ ratio was increased in the patients in whom this analysis was performed. By imaging techniques it was observed that 9 of the 16 patients presented fatty liver ( 7 by hepatic MRI). Of these 9 patients, only 3 presented hepatomegaly. 7 of 9 patients affected by the c.448G > C mutation had fatty infiltration, of which three of them presented in addition hepatomegaly.

Conclusions: There is a high prevalence of fatty liver in HFI patients and it is not related to obesity and insulin resistance. The diagnosis of fatty liver in HFI patients and, above all, the identification of new therapeutic approaches, can positively impact the quality of life of these patients.
\end{abstract}

๑) 2019 Elsevier Ltd and European Society for Clinical Nutrition and Metabolism. All rights reserved.

\footnotetext{
* Corresponding author. Unit of Metabolism, Cruces University Hospital, Biocruces Bizkaia Health Research Institute, GCV-CIBER de Enfermedades Raras (CIBERER), Plaza de Cruces s/n, Barakaldo, 48903, Spain.
}

E-mail address: olatz.villatebejarano@osakidetza.eus (O. Villate).

\section{Introduction}

The hereditary fructose intolerance (HFI; MIM 229600), caused by the deficiency of the aldolase $B$ enzyme (fructose-1,6bisphosphate aldolase) (EC 4.1.2.13) encoded by the $A L D O B$ gene (NM_000035.3), is an autosomal recessive hereditary metabolic disease with a prevalence of 1:20000 [1]. Although HFI presents a wide heterogeneity of phenotypic symptoms, the most prevalent 
clinical phenotype is liver impairment [1,2]. Aldolase B enzyme catalyzes different reactions including the reversible rupture of fructose-1-phosphate in glyceraldehyde and dihydroxyacetone phosphate and the excision of fructose-1,6-bisphosphate in two trioses, dihydroxyacetone phosphate and glyceraldehyde-3phosphate. Therefore, this enzyme plays a very important role in the control of the metabolism of fructose, as well as in the metabolism of glucose, regulating both glycolysis and gluconeogenesis.

HFI's symptoms are usually initiated with the introduction of complementary feeding in the infant, who reacts with a variety of clinical signs such as failure to thrive accompanied by vomiting, abdominal distension, jaundice and liver failure. HFI is also characterized by a set of metabolism alterations that include hypoglycemia, metabolic acidosis, hypophosphatemia, hyperuricemia, hypermagnesemia and hyperalaninemia [2] and elevated serum carbohydrate deficient transferrin (CDT) [3]. The late diagnosis of HFI associated with a continued intake of fructose leads to renal and hepatic seizures, coma and even death. The early diagnosis of HFI allows dietary treatment, consisting mainly in a diet free of fructose, sucrose and sorbitol for life.

Non-alcoholic fatty liver disease (NAFLD) is characterized by fat accumulation affecting $>5 \%$ of the liver volume that is not explained by alcohol abuse. It is the most common hepatic disorder (with $25 \%$ of prevalence worldwide). This number varies widely by geographic location and according to the methodology used for diagnosis [4-7]. NAFLD is associated with a huge clinical and economic burden [4]. This disease represents a wide spectrum of conditions ranging from fatty liver, which consists of an accumulation of fat in liver, mostly triglycerides, without inflammation or cell damage, to non-alcoholic steatohepatitis, a form of steatosis with inflammation and hepatocellular damage, which can progress to cirrhosis and liver failure, as well as liver cancer [8]. During the last decade, it has been observed that NAFLD is associated with an increase in cardiovascular risk with acceleration of arteriosclerosis and cardiovascular events [9].

It is known that fructose gives rise to NAFLD and it has recently been described that the ingestion of fructose in low amounts in aldolase B deficient mice is associated with the development of fatty liver [10]. Therefore, it is reasonable that patients with HFI present NAFLD at diagnosis, but its prevalence in patients treated and with adequate follow-up is not well documented in the literature. The aim of this study is to analyze the association between HFI and NAFLD in treated patients.

\section{Subjects and methods}

A cross-sectional observational study was conducted from February 2016 to February 2017. The population comprised genetically 16 diagnosed HFI patients from 8 Spanish centres. 10 of the patients were diagnosed in the infant period ( $0-2$ years old), 3 of them in the children period (2-14 years old) and the rest in the adult period ( $>14$ years old). We included HFI patients genetically diagnosed and in dietary treatment of fructose, sorbitol and sacarose exclusion at least for two years. Exclusion criteria were: (1) poor medical monitoring, (2) no therapeutic compliance (3), an associated to NAFLD chronic disease or transplant and/or (4) medication related to NAFLD. Patients signed the informed consent for the study.

Blood samples were collected in the morning after $8 \mathrm{~h}$ of fasting for analytical studies and anthropometric measurements were done.

\subsection{Anthropometric variables}

Recumbent length was measured with a measuring board and weight with a manual baby scale until the age of 24 months.
Thereafter, standing height was measured with a wall-mounted stadiometer and body weight, to the nearest $100 \mathrm{~g}$, with digital scales. Patients were weighed barefoot and after overnight fasting.

The nutritional status was determined by calculating the body mass index $(\mathrm{BMI})$ as BMI $=$ weight $(\mathrm{kg}) /$ height $^{2}\left(\mathrm{~m}^{2}\right)$. Patients older than 18 years old were classified as underweight (BMI < 18.5), normal weight (BMI 18.5-24.99), overweight (BMI 25-29.99), and obese (BMI $\geq 30$ ) based on the WHO criteria. Subjects less than 18 years old were classified according to BMI by using The WHO Child Growth Standards (Underweight: BMI percentile <15; Normal: BMI percentile 15 to 85 ; Overweight: BMI percentile 85 to 95 , Obese: BMI percentile $>95$ ). Absolute and normalized values were obtained through the use of the website: http://www. webpediatrica.com/endocrinoped/antropometria.php. Sex, date of birth and age at diagnosis were recorded.

\subsection{Biochemical variables}

Using standard laboratory methods, glycaemia, transaminases (ALT, AST), Gamma GT, total bilirubin, albumin, lipid profile (total cholesterol, LDL cholesterol, HDL cholesterol, triglycerides, apolipoprotein A1 and B100) and insulin were analyzed.

HOMA index was calculated by the Matthews formula: insulin $(\mu \mathrm{U} / \mathrm{mL})^{*}$ [glucose $\left.\left.(\mathrm{mmol} / \mathrm{L})\right] / 22.5\right]$. HOMA index $\geq$ a 2.5 indicates insulin resistance. QUICK index: $1 / \log 10$ basal insulin (uUI/ $\mathrm{mL})+\log 10$ basal glucose $(\mathrm{mg} / \mathrm{dL})$, which evaluates insulin sensitivity.

Study of sialotransferrins was done by HPLC technique (liquid chromatography of high efficiency or high performance liquid chromatography).

Study of methylation variables: SAM (S-adenosylmethionine), SAH (S-adenosylhomocysteine) and SAM/SAH ratio. Blood samples were collected from the antecubital vein into EDTA tubes, placed on ice and immediately centrifuged at $1000 \times \mathrm{g}$ at $4^{\circ} \mathrm{C}$ for $5 \mathrm{~min}$. Due to the instability of SAM, it is very important to use an ice bath during the process. The HPLC system consisted of an Agilent 1100 series liquid chromatograph coupled to a UV absorbance detector of the same series. SAM and SAH were separated on a Nucleosil C18 column (dimensions $12 \mathrm{~cm} \times 0.4 \mathrm{~cm}$, particle size $5 \mu \mathrm{m}$ ) (Teknokroma, Barcelona, Spain), that was kept at room temperature. The data were recorded and treated using the Chem Station software.

\subsection{Genetic studies}

Sanger sequencing of the $A L D O B$ gene was performed for each patient at the Center of Diagnosis of Molecular Diseases (Madrid, Spain).

\subsection{Image analysis}

Ultrasound and Hepatic Magnetic Resonance (MRI of 1.5 T) were performed in the corresponding collaborative hospital.

\subsection{Statistical analysis}

Statistical analysis was performed using SPSS $^{\circledR} 23.0$ for Windows (IBM, Chicago, IL). Data is reported as means (SDs) for normal continuous variables, medians and IQRs for non-normally distributed. For qualitative variables and categorical data, the relative frequency was calculated using contingency tables and chi-squared test. Statistical significance was set at the $\mathrm{p}<0.05$ level.

\subsection{Ethics}

The study protocol was approved by the Research Ethics Committee of Basque Country. Written informed consent was obtained 
from parents or legal guardians of children (below 16 years of age) and patients included.

\section{Results}

\subsection{Anthropometric characteristics}

The study was conducted on a sample of 16 patients diagnosed with fructosemia with confirmed genetic study: 5 males and 11 women aged from 3 years to 48 . $81 \%$ of the patients were younger than 18 years old. The age of diagnosis ( $0.1-45$ years) varied depending on the expression of the clinic, and based on the family study for presenting affected siblings.

Table 1 shows the characteristics of the subjects involved in this study. The subjects present a BMI lower than the Spanish average for their age comparing each individual with the expected BMI according to their age (median 17.4 in absolute values and 37.5 in percentiles) [11]. None of them presents a situation of obesity and only one of them has a BMI value at overweight levels.

\subsection{Biochemical parameters}

Table 2 shows the biochemical characteristics of the subjects. The lipid and the liver profiles show values within normality. CDT is within normal ranges.

\subsection{Genetics}

Genetic analyses of the patients revealed 4 different mutations in the $A L D O B$ gene (Fig. 1). With a total of 16 affected subjects, the mutations c.448G > C (p.Ala150Pro) and c.360_363delCAAA in homozygosis represent $44 \%$ and $37 \%$ respectively; c.448G > C

Table 1

Current anthropometrics.

\begin{tabular}{lllll}
\hline & Absolute values & Median & Percentiles & Median \\
\hline Weight $(\mathrm{Kg})$ & $11-64.4$ & 38.5 & $14-86$ & 22.5 \\
Height $(\mathrm{cm})$ & $86-166$ & 150.25 & $11-82$ & 46.5 \\
BMI $\left(\mathrm{kg} / \mathrm{m}^{2}\right)$ & $14.21-25.41$ & 17.4 & $11-92$ & 37.5 \\
\hline
\end{tabular}

Table 2

Biochemical parameters.

\begin{tabular}{llll}
\hline Parameter & $\mathrm{n}$ & Median & Range \\
\hline Lipid profile & & & \\
TOTAL CHOL mg/DI [110-240] & 14 & 191 & $149-315$ \\
HDL-CHOL mg/dL [>40] & 14 & 77 & $37-124$ \\
LDL-CHOL mg/dL [<130] & 14 & 120 & $49-179$ \\
TG mg/dL [30-170] & 14 & 70 & $39-131$ \\
APO A1 mg/dL & 9 & 202 & $165-219$ \\
APO B100 mg/dL & 9 & 91 & $67-111$ \\
Hepatic profile & & & \\
GOT/AST U/L [5-47] & 16 & 26 & $18-358$ \\
GPT/ALT U/L [5-47] & 16 & 25 & $15-438$ \\
GGT U/L [5-40] & 16 & 14 & $10-204$ \\
FA U/L [30-106] & 14 & 198 & $51-398$ \\
Albumin g/dL [3.5-5] & 13 & 4.65 & $3.4-5.1$ \\
Total Bil mg/dL [0.2-1.2] & 12 & 0.5 & $0.3-1$ \\
CDT & & & \\
Disialotransferrins \% [0,7-2,8] & 12 & $1.6 \%$ & $1-3.6$ \\
Trisialotransferrins \% [1,7-8,7] & 4 & $4.9 \%$ & $4.35-9.2$ \\
Tetrasialotransferrin \%s [60,0-84,6] & 4 & $76.4 \%$ & $68.6-77.9$ \\
Pentasialo + hexasialotransferrins \% [10,3-25,0] & 4 & $15.3 \%$ & $12.8-24.3$ \\
\hline
\end{tabular}

CHOL: cholesterol; HDL: High-Density Lipoprotein; LDL: Low-Density Lipoprotein; TG: triglycerides; APO: apolipoprotein; GOT/AST: Glutamate-Oxaloacetate Transaminase/Aspartate transaminase; GPT/ALT: Glutamate-Pyruvate Transaminase/ Alanine transaminase; GGT: Gamma-glutamyltransferase; FA: Fatty Acids; Bil: bilirubin.

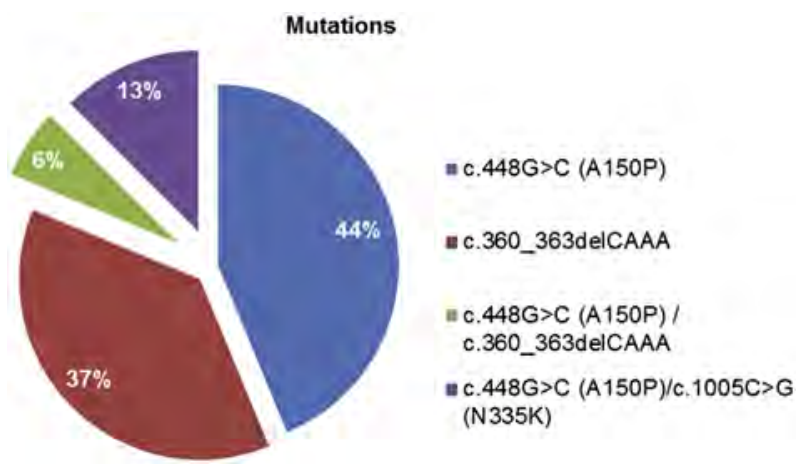

Fig. 1. Genetic mutations in the $A L D O B$ gene found in our patients. $44 \%$ of the patients in our study present the c.448G > C mutation.

(p.Ala150Pro)/c.1005C > G (p.Asn335Lys) in heterozygosis, 13\%; c.448G > C (p.Ala150Pro) and c.360_363delCAAA, both in heterozygosis, $6 \%$.

\subsection{Imaging analysis}

Using imaging techniques it was determined that 9 of the 16 patients present fatty liver ( 7 by hepatic MRI). That means that in $56 \%$ of the subjects there was at least a slight and diffuse increase in hepatic echogenicity, but periportal and diaphragmatic echogenicity was still appreciable. This corresponds to grade 1 of hepatic steatosis using established criteria [12]. Of these 9 patients, only 3 presented hepatomegaly, and none of them splenomegaly (Table 3). Statistical analysis indicates that hepatomegaly is not related to having hepatic steatosis in our patients (chi-square test 2.87, $\mathrm{p}>0.05$ ).

\subsection{Genetic and hepatic alteration}

Table 4 shows the correlation between liver lesions and the most prevalent mutations, where seven of the nine patients affected by the c.448G > C (p.Ala150Pro) mutation (78\%) had NAFLD, of which three of them presented hepatomegaly in addition. Regarding the c.360_363delCAAA mutation, only two of the seven patients had NAFLD, and none of them had hepatomegaly.

Statistical analyses revealed an association between having NAFLD and the genotype of the patient (chi-square test 3.87, $\mathrm{p}<0.05$ ).

\subsection{Methylation and insulin resistance study}

Due to the difficulties of determining the levels of SAM, SAH and SAM/SAH ratio, they were measured only in three local patients

Table 3

Nonalcoholic fatty liver.

\begin{tabular}{lllll}
\hline & Total & Percentage $\%$ & $\%<18$ years & $\%>18$ years \\
\hline Hepatic steatosis & $9 / 16$ & 56 & 77.78 & 22.22 \\
Hepatomegaly & $3 / 16$ & 19 & 66.6 & 33.4 \\
\hline
\end{tabular}

Chi-square test $2.87, \mathrm{p}>0.05$.

Table 4

Correlation between liver lesions and the most prevalent mutations.

\begin{tabular}{llll}
\hline Mutation & $\begin{array}{l}\text { Absolute } \\
\text { values }\end{array}$ & $\begin{array}{l}\text { NAFLD } \\
\text { (Yes/No) }\end{array}$ & $\begin{array}{l}\text { Hepatomegaly } \\
\text { (Yes/No) }\end{array}$ \\
\hline c.448G > C (p.Ala150Pro) & 9 & $7 / 2$ & $3 / 6$ \\
c.360_363delCAAA & 7 & $2 / 5$ & 0 \\
\hline
\end{tabular}

Chi-square test $3.87, \mathrm{p}<0.05$. 
Table 5

Levels of SAM, SAH and SAM/SAH ratio for three patients and reference range.

\begin{tabular}{lllll}
\hline & CASE 1 & CASE 2 & CASE 3 & Range (uM) \\
\hline SAM (uM) & 4.07 & 11.7 & 3.6 & $2.0-3.5$ \\
SAH (uM) & 0.09 & 0.05 & 0.05 & $0.05-0.24$ \\
SAM/SAH & 42.4 & 247.4 & 72.4 & $11.8-30.3$ \\
\hline
\end{tabular}

with hepatic steatosis (Table 5) because the shipment of the rest of the samples to analyze SAM and SAH provoked deterioration of the metabolites. SAM and SAH are very unstable so it is very important to use an ice bath during the sample preparation. Blood samples must be collected and placed on ice immediately and centrifuged.

SAM concentrations and SAM/SAH ratio were above reference values indicating the altered methylation cycle [13]. In these patients the HOMA index (0.43-1.34) and Quick index (0.37-0.45) were in normal range for our population [14].

\section{Discussion}

Our data confirm the high prevalence of the c.448G > C (p.Ala150Pro) pathogenic variant in our patients (44\%) as previously reported in other large European and USA cohorts $[15,16]$. The mutation is a $\mathrm{G} \rightarrow \mathrm{C}$ change in exon 5 that creates a new recognition site for the restriction enzyme Ahall and results in an amino acid substitution (Ala $\rightarrow$ Pro) at position 150 of the protein within a critical region for substrate binding. This could explain that almost $78 \%$ of our patients with the c.448G > C (p.Ala150Pro) variant presented the fatty infiltration, unlike the other mutations, which, despite representing almost $40 \%$ of the sample, were not associated with NAFLD. In addition, hepatomegaly has been found in three patients with this mutation.

NAFLD is traditionally associated with patients with hereditary intolerance to fructose with poor metabolic control and a high BMI, however, in our analysis all patients who have been studied have followed adequate metabolic control and their BMI is within normal range. This allows us to establish a strong relationship between NAFLD and the treated fructosemia, since more than half of the patients had this hepatic alteration.

Ultrasonography is the most inexpensive and widely available imaging test for NAFLD. Typical sonographic findings of NAFLD are hepatomegaly and increased echogenicity. Unfortunately, ultrasonography is not sensitive if less than $30 \%$ of the liver is involved by steatosis. Variability in operator skill and the limiting body habitus of the typical NAFLD patient can lead to inadequate or inconsistent results. Although changes of cirrhosis and portal hypertension may be identified on ultrasound, it is neither a sensitive nor specific modality for this diagnosis. Given these limitations, other imaging studies are being investigated for the diagnosis and risk stratification of NAFLD patients. Magnetic resonance imaging, including magnetic resonance spectroscopy, has shown good sensitivity and specificity in detecting and quantifying steatosis [17]. In our study, the vast majority of the NAFLD diagnosis was done by hepatic MRI, but its percentage can be under-diagnosed because many of the patients only had the ultrasonography as image test.

Currently there are no data on the physiopathological mechanisms that produce fatty liver in patients with HFI. Previous studies have established a clear relationship between the accumulation of fat in the liver and the inhibition of the protein kinase activated by AMP (AMPK), both in animal models of obesity [18] and in human metabolic diseases such as the insulin resistance, obesity and sedentary life [19]. AMPK is a protein regulated by posttranslational modifications like phosphorylation and also allosterically regulated through the competitive union of ATP, so that the AMPK often functions as a sensor of the ratio between AMP/ATP and/or ADP/ ATP, which reflect cellular energy levels. Thus, it has been described that the activation of AMPK leads to the inhibition of energyconsuming anabolic pathways, such as the synthesis of fatty acids and sterols, and the activation of catabolic pathways that produce energy, such as the $\beta$-oxidation of fatty acids [20].

On the other hand, the accumulation of fructose-1-phosphate in HFI patients with aldolase B deficit carries a depletion of free inorganic phosphate [21]. This decrease in the reserve of cellular inorganic phosphate activates the enzyme AMP deaminase [22]. It has been documented in HFI patients, using magnetic resonance spectroscopy, that the accumulation of hepatic fructose-1phosphate and the decrease in hepatic ATP concentration between 40 and $60 \%$ after infusion of $200 \mathrm{mg} / \mathrm{kg}$ of fructose, is so small that it did not even increase the concentration of uric acid above the values of normality $[23,24]$. In addition, it has been found that some drugs that activate AMPK act through inhibition of the AMP deaminase [25]. These evidences suggest that in HFI patients, the accumulation of fructose-1-phosphate and the decrease in associated free inorganic phosphate may inhibit the activation of AMPK, through the activation of the deaminase AMP, leading to a decrease in the $\beta$-oxidation of fatty acids, resulting in the accumulation of fatty acids in the form of triglycerides in the liver.

Lipidomic studies have revealed that NAFLD is characterized by an increase in lipogenesis, and the activity of desaturases and lipoxygenase [26]. Other metabolic disturbances described in NAFLD are changes in the proportion of phosphatidylcholine and phosphatidylethanolamine $[27,28]$.

In recent years, a restriction of dietary methionine intake to approximately $2 \mathrm{mg} / \mathrm{kg} \mathrm{BW} / \mathrm{d}$ has been associated with an increase in hepatic $\beta$-oxidation in obese adults with metabolic syndrome [29]. The same association was observed in animal models of obesity with a $0.12 \%$ methionine restriction diet [30]. Methionine is an essential amino acid, component of the methylation cycle, a major regulator of detoxification, inflammation, energy production, and DNA maintenance processes among others. It has also been observed that changes in the methylation index, quantified by the ratio of the intermediaries S-adenosyl-methionine (SAM) and Sadenosyl-L-homocysteine (SAH), are directly associated with a deregulation of the export of hepatic lipids mediated by very low density lipoproteins (VLDL), which are responsible for transporting triglycerides, cholesterol esters and phospholipids from the liver to the extrahepatic tissues [31]. The increase in methionine in blood is considered a warning symptom for the diagnosis of HFI [32]. Although in our work a small number of HFI patients with fatty liver have been studied, we have observed an increase in the ratio $\mathrm{SAM} / \mathrm{SAH}$ that is associated with changes in the methylation cycle. Recent studies have described the beneficial effects of a methionine limited diet, with a very important role in regulating the methylation cycle, so that this diet increases hepatic $\beta$-oxidation, resulting in a decrease in the levels of phosphatidylethanolamine in the VLDL-TG long molecules enriched in apolipoprotein $\mathrm{E}$.

It should be considered that a limited diet in methionine could be beneficial for the prevention and/or treatment of fatty liver in HFI patients, acting at two levels, increasing the beta-oxidation of fatty acids and optimizing the lipids transport to extrahepatic tissues. We must have in mind that the dietary treatment of these patients favors a high protein diet.

It is important to emphasize that fatty liver often evolves to steatohepatitis, hepatic fibrosis, cirrhosis and hepatic failure, and is associated with an increased risk of cardiovascular disease, diseases associated to a great morbidity and mortality. Therefore, the diagnosis of fatty liver in HFI patients and, above all, the identification of new therapeutic approaches, can positively impact the quality of life of these patients. 
This study has some limits that reduce the power of the results: first it is a retrospective study that looks at patients at different ages and stages of disease; therefore we cannot be entirely certain of the clinical course of NAFLD. Second, the limited sample size precludes proper statistical analysis and a prospective and larger study is necessary to better clarify the evolution of the disease. Nevertheless, we believe that our study enriches the knowledge of the pathologic liver changes in hereditary fructose intolerance disease. Obviously, more functional data and a longer follow-up are necessary to better understand the pathophysiological mechanism of NAFLD in this genetic disorder in order to develop novel therapeutic approaches.

\section{Statement of authorship}

LAE, MLC, CA, IV, MB, JB, MCG, MR, RS and JDH collected data and reviewed the manuscript. JDH contributed to manuscript writing. RS contributed to the statistical analysis. LAE, FA and OV designed the study, carried out the statistical analysis, drafted the initial manuscript and reviewed and revised the manuscript. All authors approved the final manuscript.

\section{Funding sources}

This research did not receive any specific grant from funding agencies in the public, commercial, or not-for-profit sectors.

\section{Conflict of interest statement}

The authors declare no conflict of interest.

\section{Acknowledgements}

Authors would like to thank patients for their collaboration in our study.

\section{References}

[1] Cross NCP, Cox TM, de Franchis R, Sebastio G, Dazzo C, Tolan DR, et al. Mo lecular analysis of aldolase B genes in hereditary fructose intolerance. Lancet 1990;335(8685):306-9.

[2] Baker P, Ayres L, Gaughan S, Weisfeld-Adams J, editors. Hereditary Fructose Intolerance, Source: GeneReviews ${ }^{\circledR}$ [Internet]. Seattle (WA): University of Washington; 1993-2017. Seattle.

[3] Quintana E, Sturiale L, Montero R, Andrade F, Fernandez C, Couce ML, et al. Secondary disorders of glycosylation in inborn errors of fructose metabolism. J Inherit Metab Dis 2009;32:S273-8.

[4] Younossi ZM, Koenig AB, Abdelatif D, Fazel Y, Henry L, Wymer M. Global epidemiology of nonalcoholic fatty liver disease-Meta-analytic assessment of prevalence, incidence, and outcomes. Hepatology 2016;64(1):73-84.

[5] Wong RJ, Ahmed A. Obesity and non-alcoholic fatty liver disease: disparate associations among Asian populations. World J Hepatol 2014 May 27;6(5): 263-73.

[6] Lewis JR, Mohanty SR. Nonalcoholic fatty liver disease: a review and update. Dig Dis Sci 2010 Mar:55(3):560-78.

[7] Torres DM, Harrison SA. Diagnosis and therapy of nonalcoholic steatohepatitis. Gastroenterology May 2008;134(6):1682-98.

[8] Michelotti GA, Machado MV, Diehl AM. NAFLD, NASH and liver cancer. Nat Rev Gastroenterol Hepatol 2013;10(11):656-65.

[9] Lonardo A, Nascimbeni F, Mantovani A, Targher G. Hypertension, diabetes, atherosclerosis and NASH: cause or consequence? J Hepatol 2018;68(2):335-52.

[10] Oppelt SA, Sennott EM, Tolan DR. Aldolase-B knockout in mice phenocopies hereditary fructose intolerance in humans. Mol Genet Metabol 2015;114(3): 445-50.
[11] López de Lara D, Santiago Paniagua P, Tapia Ruiz M, Rodríguez Mesa MD, Gracia Bouthelier R, Carrascosa Lezcano A. Valoración del peso, talla e IMC en niños, adolescentes y adultos jóvenes de la Comunidad Autónoma de Madrid. An Pediatr 2010;73(6):305-19.

[12] Sanyal AJ. AGA technical review on nonalcoholic fatty liver disease. Gastroenterology 2002;123(5):1705-25.

[13] Andrade F, Rodríguez-Soriano J, Prieto JA, Aguirre M, Ariceta G, Lage S, et al. Methylation cycle, arginine-creatine pathway and asymmetric dimethylarginine in paediatric renal transplant. Nephrol Dial Transplant 2011;26(1): $328-36$.

[14] García Cuartero B, García Lacalle C, Jiménez Lobo C, González Vergaz A, Calvo Rey C, Alcázar Villar MJ, et al. Índice HOMA y QUICKI, insulina y péptido C en niños sanos. Puntos de corte de riesgo cardiovascular. An Pediatr 2007;66(5): 481-90.

[15] Caciotti A, Donati MA, Adami A, Guerrini R, Zammarchi E, Morrone A. Different genotypes in a large Italian family with recurrent hereditary fructose intolerance. Eur J Gastroenterol Hepatol 2008;20(2):118-21.

[16] Coffee EM, Yerkes L, Ewen EP, Zee T, Tolan DR. Increased prevalence of mutant null alleles that cause hereditary fructose intolerance in the American population. J Inherit Metab Dis 2010;33(1):33-42.

[17] Spengler EK, Loomba R. Recommendations for diagnosis, referral for liver biopsy, and treatment of nonalcoholic fatty liver disease and nonalcoholic steatohepatitis. Mayo Clin Proc 2015;90(9):1233-46.

[18] Woods A, Williams JR, Muckett PJ, Mayer FV, Liljevald M, Bohlooly YM, et al. Liver-specific activation of AMPK prevents steatosis on a high-fructose diet. Cell Rep 2017;18(13):3043-51.

[19] Ruderman N, Prentki M. Erratum: AMP kinase and malonyl-CoA: targets for therapy of the metabolic syndrome. Nat Rev Drug Discov 2004;3(4): 340-51.

[20] Cool B, Zinker B, Chiou W, Kifle L, Cao N, Perham M, et al. Identification and characterization of a small molecule AMPK activator that treats key components of type 2 diabetes and the metabolic syndrome. Cell Metabol 2006;3(6): 403-16.

[21] Lanaspa MA, Sanchez-Lozada LG, Cicerchi C, Li N, Roncal-Jimenez CA, Ishimoto T, et al. Uric acid stimulates fructokinase and accelerates fructose metabolism in the development of fatty liver. PLoS One 2012;7(10): e47948.

[22] Van den Berghe H, Bruntman M, Vannestes R, Hers HG. The mechanism of adenine triphosphate depletion in the liver after a load of fructose. A kinetic study of liver adenylate deaminase. Biochem J 1977;134:637-45.

[23] Boesiger P, Buchli R, Meier D, Steinmann B, Gitzelmann R. Changes of liver metabolite concentrations in adults with disorders of fructose metabolism after intravenous fructose by 31P magnetic resonance spectroscopy. Pediatr Res 1994;36(4):436-40.

[24] Oberhaensli R, Taylor D, Rajagopalan B, Radda G, Collins J, Leonard J, et al. Study of hereditary fructose intolerance by use of 31P magnetic resonance spectroscopy. Lancet 1987;330(8565):931-4.

[25] Ouyang J, Parakhia RA, Ochs RS. Metformin activates AMP kinase through inhibition of AMP deaminase. J Biol Chem 2011;286(1):1-11.

[26] Puri P, Wiest MM, Cheung O, Mirshahi F, Sargeant C, Min HK, et al. The plasma lipidomic signature of nonalcoholic steatohepatitis. Hepatology 2009;50(6): 1827-38.

[27] Jacobs RL, van der Veen JN, Vance DE. Finding the balance: the role of Sadenosylmethionine and phosphatidylcholine metabolism in development of nonalcoholic fatty liver disease. Hepatology 2013;58(4):1207-9.

[28] Martinez-Una M, Varela-Rey M, Cano A, Fernández-Ares L, Beraza N, Aurrekoetxea I, et al. Excess S-adenosylmethionine reroutes phosphatidylethanolamine towards phosphatidylcholine and triglyceride synthesis. Hepatology 2013;58(4):1296-305.

[29] Plaisance EP, Greenway FL, Boudreau A, Hill KL, Johnson WD, Krajcik RA, et al. Dietary methionine restriction increases fat oxidation in obese adults with metabolic syndrome. J Clin Endocrinol Metabol 2011;96. E836.40.

[30] Malloy VL, Perrone CE, Mattocks DAL, Ables GP, Caliendo NS, Orentreich DS, et al. Methionine restriction prevents the progression of hepatic steatosis in leptin-deficient obese mice. Metabolism 2013:62(11):1651-61.

[31] Martínez-Uña M, Varela-Rey M, Mestre D, Fernández-Ares L, Fresnedo O, Fernandez-Ramos D, et al. S-Adenosylmethionine increases circulating verylow density lipoprotein clearance in non-alcoholic fatty liver disease. J Hepatol 2015:62(3):673-81.

[32] Steinmann B, Gitzelmann R, Van den Berghe G. Disorders of fructose metabolism. In: Valle D, Beaudet AL, Vogelstein B, Kinzler KW, Antonarakis SE, Ballabio A, et al., editors. The online metabolic and molecular bases of inherited disease. New York, NY: McGraw-Hill; 2014. http://ommbid. mhmedical.com/content.aspx?bookid=971\&sectionid=62671933. 